\title{
TIME DOMAIN BOUNDARY ELEMENT METHODS FOR THE NEUMANN PROBLEM: ERROR ESTIMATES AND ACOUSTIC PROBLEMS*
}

\author{
Heiko Gimperlein \\ Maxwell Institute for Mathematical Sciences and Department of Mathematics, \\ Heriot-Watt University, Edinburgh EH14 4AS, UK, and \\ Institute for Mathematics, University of Paderborn, 33098 Paderborn, Germany \\ Email: h.gimperlein@hw.ac.uk \\ Ceyhun Özdemir and Ernst P. Stephan \\ Institute of Applied Mathematics, Leibniz University Hannover, 30167 Hannover, Germany \\ Email: oezdemir@ifam.uni-hannover.de,stephan@ifam.uni-hannover.de
}

\begin{abstract}
We investigate time domain boundary element methods for the wave equation in $\mathbb{R}^{3}$, with a view towards sound emission problems in computational acoustics. The Neumann problem is reduced to a time dependent integral equation for the hypersingular operator, and we present a priori and a posteriori error estimates for conforming Galerkin approximations in the more general case of a screen. Numerical experiments validate the convergence of our boundary element scheme and compare it with the numerical approximations obtained from an integral equation of the second kind. Computations in a half-space illustrate the influence of the reflection properties of a flat street.
\end{abstract}

Mathematics subject classification: 65N38, 65R20, 74J05.

Key words: Time domain boundary element method, Wave equation, Neumann problem, Error estimates, Sound radiation.

\section{Introduction}

Motivated by the sound radiation of tires [2], this article analyzes time domain boundary element methods for a scattering or emission problem for the wave equation outside a soundhard obstacle.

Let $d \geq 2$ and $\Omega^{i} \subset \mathbb{R}^{d}$ be a bounded Lipschitz domain. We aim to find a weak solution to an acoustic initial boundary problem for the wave equation in $\Omega^{e}=\mathbb{R}^{d} \backslash \overline{\Omega^{i}}$ :

$$
\begin{array}{ll}
\frac{\partial^{2} u}{\partial t^{2}}-\Delta u=0 & \text { in } \mathbb{R}^{+} \times \Omega^{e}, \\
u(0, x)=\frac{\partial u}{\partial t}(0, x)=0 & \text { in } \Omega^{e}, \\
\frac{\partial u}{\partial n}=\tilde{g} & \text { on } \mathbb{R}^{+} \times \Gamma .
\end{array}
$$

Here $n$ denotes the inward unit normal vector to $\Gamma=\partial \Omega^{e}$, and $2 \tilde{g}=g$ lies in a suitable Sobolev space.

\footnotetext{
* Received January 4, 2016 / Revised version received June 27, 2016 / Accepted October 18, 2016 /

Published online October 11, 2017 /
} 
This article reduces the boundary problem (1.1) to a time dependent integral equation on $\mathbb{R}^{+} \times \Gamma$ and studies Galerkin time domain boundary element methods for its approximation. While we focus on the hypersingular integral equation, numerical examples compare it to an integral equation of the second kind.

Time domain boundary integral formulations for hyperbolic equations and their numerical solution were introduced by Friedman and Shaw [7], resp. Cruse and Rizzo [4]. A first mathematical analysis of time dependent boundary element methods goes back to Bamberger and Ha-Duong [1,12], see also [9] for Dirichlet and acoustic boundary problems in a half-space. First numerical experiments for integral equations of the second kind in the full space were reported by Ding et al. [5], and the practical realization of the numerical marching-on-in-time scheme include the Ph.D. thesis of Terrasse [19] as well as [14]. Also, fast collocation methods have been developed in the engineering literature [21]. Some recent work around space-time adaptive methods and applications is surveyed in [8]. A detailed exposition of the mathematical background of time domain integral equations and their discretizations is available in the lecture notes by Sayas [18].

In this work we investigate the Neumann problem (1.1), present a priori and a posteriori error estimates for the Galerkin solution of the time dependent hypersingular integral equation of the first kind (with the normal derivative of the double layer potential). We compare the numerical scheme for the hypersingular equation with numerical approximations of an integral equation of the second kind (with the normal derivative of the single layer potential). We analyze the integral equations in the more general setting of a screen $\Gamma$, i.e., allow $\partial \Gamma \neq \emptyset$, which will prove relevant for work in progress on dynamic contact problems.

A motivation for these results comes from applications to traffic noise $[2,9,10]$, where adaptive methods based on a posteriori error estimates are crucial to resolve singular geometries. With this application in mind, we also present numerical results in an acoustic half-space. Here, $\Omega^{i} \subset \mathbb{R}_{+}^{d}$ is a bounded domain with $\mathbb{R}_{+}^{d} \backslash \overline{\Omega^{i}}$ Lipschitz, and the Neumann boundary conditions on $\Gamma=\partial \Omega^{i} \cap \mathbb{R}_{+}^{d}$ are supplemented by acoustic boundary conditions

$$
\frac{\partial u}{\partial n}-\alpha \frac{\partial u}{\partial t}=0
$$

on $\mathbb{R}^{d-1} \times\{0\}=\partial \mathbb{R}_{+}^{d}, \alpha \geq 0$. Screens arise naturally when $\partial \Omega^{i} \cap \partial \mathbb{R}_{+}^{d} \neq \emptyset$.

Notation: To simplify notation, we will write $f \lesssim g$, if there exists a constant $C>0$ independent of the arguments of the functions $f$ and $g$ such that $f \leq C g$. We will write $f \lesssim_{\sigma} g$, if $C$ may depend on $\sigma$.

\section{Time-domain Integral Equations and Discretization}

\subsection{Boundary integral equations}

Space-time anisotropic Sobolev spaces on the boundary $\Gamma$ provide a convenient setting to study the mapping properties of the time-dependent layer operators [3,13]. We more generally consider the case of a screen, where the orientable, $(d-1)$-dimensional Lipschitz submanifold $\Gamma \subset \mathbb{R}^{d}$ may have a boundary. If $\partial \Gamma \neq \emptyset$, first extend $\Gamma$ to a closed, orientable manifold $\widetilde{\Gamma}$.

For $\sigma>0, s, r \in \mathbb{R}$ the space $H_{\sigma}^{s}\left(\mathbb{R}^{+}, H^{r}(\widetilde{\Gamma})\right)$ consists of certain distributions $\phi$ on $\mathbb{R}^{+} \times \widetilde{\Gamma}$, vanishing at $t=0$, such that in local coordinates the space-time Fourier-Laplace transform $\mathcal{F} \phi$ 
satisfies

$$
\|\phi\|_{s, r, \widetilde{\Gamma}}=\left(\iint|\omega+i \sigma|^{2 s}\left(|\omega+i \sigma|^{2}+|\xi|^{2}\right)^{r}|\mathcal{F} \phi(\omega+i \sigma, \xi)|^{2} d \xi d \omega\right)^{\frac{1}{2}}<\infty .
$$

The space $H_{\sigma}^{s}\left(\mathbb{R}^{+}, \widetilde{H}^{r}(\Gamma)\right)$ is then defined as the closed subspace of distributions $\phi \in H_{\sigma}^{s}\left(\mathbb{R}^{+}\right.$, $\left.H^{r}(\widetilde{\Gamma})\right)$ with support in $\bar{\Gamma}$, and $H_{\sigma}^{s}\left(\mathbb{R}^{+}, H^{r}(\Gamma)\right)$ as the quotient space $H_{\sigma}^{s}\left(\mathbb{R}^{+}, H^{r}(\widetilde{\Gamma})\right) / H_{\sigma}^{s}\left(\mathbb{R}^{+}\right.$, $\left.H^{r}(\widetilde{\Gamma} \backslash \bar{\Gamma})\right)$. The corresponding norms are denoted by $\|\phi\|_{s, r, \Gamma, *}$ resp. $\|\phi\|_{s, r, \Gamma}$. By truncation, we also obtain anisotropic Sobolev spaces on finite time-intervals $[0, T], H_{\sigma}^{s}\left([0, T], \widetilde{H}^{r}(\Gamma)\right)$ and $H_{\sigma}^{s}\left([0, T], H^{r}(\Gamma)\right)$. When $r \in \frac{1}{2} \mathbb{Z}$, resp. $s+r \in \frac{1}{2} \mathbb{Z}$, there are subtle distinctions between the spaces of supported and extensible distributions, and the closure of $C_{0}^{\infty}$, as is known for time-independent screen problems. See $[9,13]$ for a more detailed discussion.

Layer operators allow to reduce the boundary problem (1.1) to an integral equation on the boundary $\Gamma$, both in the case of the whole space $\mathbb{R}^{d}$ and in the half-space with acoustic boundary conditions (1.2). These operators are based on a Green's function $G$ for the wave equation. In $\mathbb{R}^{3}, G$ is explicitly given by

$$
G(t-s, x, y)=\frac{\delta\left(t-s-r\left(y_{3}\right)\right)}{4 \pi r\left(y_{3}\right)},
$$

and in $\mathbb{R}_{+}^{3}$ by $[15]$

$$
G(t-s, x, y)=\frac{\delta\left(t-s-r\left(y_{3}\right)\right)}{4 \pi r\left(y_{3}\right)}+\frac{\delta\left(t-s-r\left(-y_{3}\right)\right)}{4 \pi r\left(-y_{3}\right)}+\Sigma,
$$

with

$$
\Sigma=\frac{\alpha}{2 \pi} \frac{\partial}{\partial t} \frac{H\left(t-s-r\left(-y_{3}\right)\right)}{\sqrt{\left(t-s+\alpha\left(x_{3}+y_{3}\right)\right)^{2}+\left(\alpha^{2}-1\right) R^{2}}} .
$$

Here $H$ denotes the Heaviside function, $R^{2}=\left(x_{1}-y_{1}\right)^{2}+\left(x_{2}-y_{2}\right)^{2}$ and $r\left( \pm y_{3}\right)^{2}=R^{2}+\left(x_{3} \mp\right.$ $\left.y_{3}\right)^{2}$. The second and third terms on the right-hand side of $G$ represent the field reflected by the plane $\partial \mathbb{R}_{+}^{3}$.

From a single layer potential ansatz for the solution $u$ of (1.1):

$$
u(t, x)=\int_{\mathbb{R}^{+} \times \Gamma} G(t-\tau, x, y) \varphi(\tau, y) d \tau d s_{y}
$$

with $\varphi(s, y)=0$ for $s \leq 0$, one obtains an equivalent boundary integral equation of the second kind for the unknown density $\varphi$ on $\Gamma$ :

$$
\left(-I d+K^{\prime}\right) \varphi=2 \frac{\partial u}{\partial n}=g .
$$

Here, the time-dependent adjoint double layer operator $K^{\prime}$ is defined by

$$
K^{\prime} \varphi(t, x)=2 \int_{\mathbb{R}^{+} \times \Gamma} \frac{\partial G}{\partial n_{x}}(t-\tau, x, y) \varphi(\tau, y) d \tau d s_{y} .
$$

Knowing $\varphi$, one reconstructs the solution $u$ of the wave equation from (2.2). Numerical schemes based on (2.3) have been explored in [2]. However, little is known about the theoretical analysis for discretizations of time dependent integral equations of the second kind. 
In this article we focus on an integral equation of the first kind, which we obtain from a double layer potential ansatz for $u$ :

$$
u(t, x)=\int_{\mathbb{R}^{+} \times \Gamma} \frac{\partial G}{\partial n_{y}}(t-\tau, x, y) \psi(\tau, y) d \tau d s_{y}
$$

with $\psi(s, y)=0$ for $s \leq 0$. The wave equation (1.1) is then equivalent to a time-dependent hypersingular equation for the unknown density $\psi$ on $\Gamma$ :

$$
W \psi=2 \frac{\partial u}{\partial n}=g
$$

where the time-dependent hypersingular operator $W$ for the half-space is given by

$$
W \psi(t, x)=2 \int_{\mathbb{R}^{+} \times \Gamma} \frac{\partial^{2} G}{\partial n_{x} \partial n_{y}}(t-\tau, x, y) \psi(\tau, y) d \tau d s_{y} .
$$

More generally than for $\Gamma=\partial \Omega$, we consider the integral equations (2.3) and (2.6) on an orientable, $(d-1)$-dimensional Lipschitz submanifold $\Gamma \subset \mathbb{R}^{d}$ with boundary. For the analysis we recall the mapping and coercivity properties of $K^{\prime}$ and $W$ :

Theorem 2.1. a) The following operators are continuous for $r \in \mathbb{R}$ :

$$
\begin{array}{ll}
K^{\prime}: & H_{\sigma}^{r+1}\left(\mathbb{R}^{+}, \widetilde{H}^{-\frac{1}{2}}(\Gamma)\right) \rightarrow H_{\sigma}^{r}\left(\mathbb{R}^{+}, H^{-\frac{1}{2}}(\Gamma)\right), \\
W: & H_{\sigma}^{r+1}\left(\mathbb{R}^{+}, \widetilde{H}^{\frac{1}{2}}(\Gamma)\right) \rightarrow H_{\sigma}^{r}\left(\mathbb{R}^{+}, H^{-\frac{1}{2}}(\Gamma)\right) .
\end{array}
$$

b) The operator $W \partial_{t}$ is weakly coercive:

$$
\int_{\mathbb{R}^{+} \times \Gamma} e^{-2 \sigma t}(W \psi(t, x)) \partial_{t} \psi(t, x) d t d s_{x} \gtrsim_{\sigma}\|\psi\|_{0, \frac{1}{2}, \Gamma, *}^{2} \cdot
$$

See [13] for part a) when $\partial \Gamma=\emptyset$. In this case part b) follows from Eq. (2.14), p. 174 in [12]. For the half-space or when $\partial \Gamma \neq \emptyset, a)$ is shown in [9]; the proof of $b$ ) is obtained by extending Ha Duong's proof in [13] for $\partial \Gamma=\emptyset$, using the modifications from [9].

The mapping and coercivity properties give a basic well-posedness theorem for the integral equations (2.3) and (2.6).

Theorem 2.2. Let $g \in H_{\sigma}^{s+2}\left(\mathbb{R}^{+}, H^{-\frac{1}{2}}(\Gamma)\right)$.

a) There exists a unique solution $\varphi \in H_{\sigma}^{s}\left(\mathbb{R}^{+}, \widetilde{H}^{-\frac{1}{2}}(\Gamma)\right)$ to (2.3). It satisfies

$$
\|\varphi\|_{s,-\frac{1}{2}, \Gamma, *} \leq C\|g\|_{s+2,-\frac{1}{2}, \Gamma}
$$

for some constant $C$ independent of $g$.

b) There exists a unique solution $\psi \in H_{\sigma}^{s+1}\left(\mathbb{R}^{+}, \widetilde{H}^{\frac{1}{2}}(\Gamma)\right)$ to (2.6). It satisfies

$$
\|\psi\|_{s+1, \frac{1}{2}, \Gamma, *} \leq C\|g\|_{s+2,-\frac{1}{2}, \Gamma}
$$

for some constant $C$ independent of $g$.

The proof of part a) uses the equivalence of (2.3) with the original PDE problem (1.1). It does not imply the well-posedness of the discretized problem. Part b) is a direct consequence of the weak coercivity estimate in Theorem 2.1b); note that the solution is less regular in time 
than the mapping properties in Theorem 2.1a) might suggest, because coercivity only holds in a weaker norm.

Both formulations, (2.3) and (2.6), will be discretized from their variational formulations, which admit a unique solution when $g \in H_{\sigma}^{\frac{5}{2}}\left(\mathbb{R}^{+}, H^{-\frac{1}{2}}(\Gamma)\right)$, resp. $g \in H_{\sigma}^{2}\left(\mathbb{R}^{+}, H^{-\frac{1}{2}}(\Gamma)\right)$, i.e. for sufficiently smooth functions of time. They are given as:

- Find $\varphi \in H_{\sigma}^{\frac{1}{2}}\left(\mathbb{R}^{+}, \widetilde{H}^{-\frac{1}{2}}(\Gamma)\right)$ such that for all $\Psi \in H_{\sigma}^{\frac{1}{2}}\left(\mathbb{R}^{+}, \widetilde{H}^{\frac{1}{2}}(\Gamma)\right)$ there holds:

$$
\int_{\mathbb{R}^{+} \times \Gamma} e^{-2 \sigma t}\left(-I d+K^{\prime}\right) \varphi \Psi d t d s_{x}=\int_{\mathbb{R}^{+} \times \Gamma} e^{-2 \sigma t} g \Psi d t d s_{x} .
$$

- Find $\psi \in H_{\sigma}^{1}\left(\mathbb{R}^{+}, \widetilde{H}^{\frac{1}{2}}(\Gamma)\right)$ such that for all $\Psi \in H_{\sigma}^{1}\left(\mathbb{R}^{+}, \widetilde{H}^{\frac{1}{2}}(\Gamma)\right)$ there holds:

$$
\int_{\mathbb{R}^{+} \times \Gamma} e^{-2 \sigma t}(W \psi) \partial_{t} \Psi d t d s_{x}=\int_{\mathbb{R}^{+} \times \Gamma} e^{-2 \sigma t} g \partial_{t} \Psi d t d s_{x} .
$$

Because of the coercivity in Theorem 2.1b), the Galerkin scheme (2.8) admits a unique solution and is stable in the norm of the space $H_{\sigma}^{0}\left(\mathbb{R}^{+}, \widetilde{H}^{\frac{1}{2}}(\Gamma)\right)$.

\subsection{Discretization}

We consider dimensions $d=2$ and 3 . If $\Gamma$ is not polygonal we approximate it by a piecewise polygonal curve resp. surface and write $\Gamma$ again for the approximation. For simplicity, when $d=3$ we will use here a surface composed of $N$ triangular facets $\Gamma_{i}$ such that $\Gamma=\cup_{i=1}^{N} \Gamma_{i}$. When $d=2$, we assume $\Gamma=\cup_{i=1}^{N} \Gamma_{i}$ is composed of line segments $\Gamma_{i}$. In each case, the elements $\Gamma_{i}$ are closed with $\operatorname{int}\left(\Gamma_{i}\right) \neq \emptyset$, and for distinct $\Gamma_{i}, \Gamma_{j} \subset \Gamma$ the intersection $\operatorname{int}\left(\Gamma_{i}\right) \cap \operatorname{int}\left(\Gamma_{j}\right)=\emptyset$.

For the time discretization we consider a uniform decomposition of the time interval $\mathbb{R}^{+}$ into subintervals $I_{n}=\left(t_{n-1}, t_{n}\right]$ with time step $\left|I_{n}\right|=\Delta t$, such that $t_{n}=n \Delta t(n=0,1, \ldots)$.

Let $\mathbb{P}^{p}$ be the space of polynomials of degree at most $p$. We choose a basis $\varphi_{1}^{p}, \cdots, \varphi_{N_{s}}^{p}$ of the space

$$
\left.V_{h}^{p}=\left\{\phi: \Gamma \rightarrow \mathbb{R}:\left.\phi\right|_{\Gamma_{i}} \in \mathbb{P}^{p} \quad \forall i \text { (and } \phi \text { continuous and }\left.\phi\right|_{\partial \Gamma}=0 \text { if } p \geq 1\right)\right\}
$$

of piecewise polynomials in space and a basis $\beta^{1, q}, \cdots, \beta^{N_{t}, q}$ of the space

$$
V_{\Delta t}^{q}=\left\{\phi: \mathbb{R}^{+} \rightarrow \mathbb{R}:\left.\phi\right|_{I_{n}} \in \mathbb{P}^{q} \quad \forall n \quad(\text { and } \phi \text { continuous and } \phi(0)=0 \text { if } q \geq 1)\right\}
$$

of piecewise polynomials in time.

Let $\mathcal{T}_{S}=\left\{T_{1}, \cdots, T_{N_{s}}\right\}$ be the spatial mesh for $\Gamma$ and $\mathcal{T}_{T}=\left\{\left(0, t_{1}\right],\left(t_{1}, t_{2}\right], \cdots,\left(t_{N_{t}-1}, T\right]\right\}$ the time mesh for a finite subinterval $(0, T]$.

We consider the tensor product of the approximation spaces in space and time, $V_{h}^{p}$ and $V_{\Delta t}^{q}$, associated to the space-time mesh $\mathcal{T}_{S, T}=\mathcal{T}_{S} \times \mathcal{T}_{T}$, and we write

$$
V_{h, \Delta t}^{p, q}=V_{h}^{p} \otimes V_{\Delta t}^{q} .
$$

These approximation spaces lead to Galerkin formulations for (2.7) and (2.8). They are given in terms of the discretized right hand sides $g_{h, \Delta t}$, resp. $\left(\partial_{t} g\right)_{h, \Delta t}$ as:

- Find $\varphi_{h, \Delta t} \in V_{h, \Delta t}^{p, q}$ such that for all test functions $\Psi_{h, \Delta t} \in V_{h, \Delta t}^{p, q}$ there holds:

$$
\int_{\mathbb{R}^{+} \times \Gamma} e^{-2 \sigma t}\left(-I+K^{\prime}\right) \varphi_{h, \Delta t} \Psi_{h, \Delta t} d t d s_{x}=\int_{\mathbb{R}^{+} \times \Gamma} e^{-2 \sigma t} g_{h, \Delta t} \Psi_{h, \Delta t} d t d s_{x} .
$$


- Find $\psi_{h, \Delta t} \in V_{h, \Delta t}^{p, q}$ such that for all test functions $\Psi_{h, \Delta t} \in V_{h, \Delta t}^{p, q}$ there holds:

$$
\int_{\mathbb{R}^{+} \times \Gamma} e^{-2 \sigma t}\left(W \psi_{h, \Delta t}\right) \partial_{t} \Psi_{h, \Delta t} d t d s_{x}=\int_{\mathbb{R}^{+} \times \Gamma} e^{-2 \sigma t} g_{h, \Delta t} \partial_{t} \Psi_{h, \Delta t} d t d s_{x} .
$$

\section{Error Estimates for the Hypersingular Integral Equation}

\subsection{An a priori error estimate}

Our first error estimate proves the convergence of the Galerkin method (2.11) if the exact solution is sufficiently smooth and the discretization is based on piecewise polynomials of sufficiently high order. In the numerical experiments in Section 5 , we shall observe convergence for more practical discretizations. See also [12], p. 182, Thm. 3, for a similar statement for closed manifolds $\Gamma \subset \mathbb{R}^{d}$ without proof.

As ingredient, we require an inverse estimate like (3.182) in [11], namely

$$
\left\|\phi_{h, \Delta t}\right\|_{1, \frac{1}{2}, \Gamma, *} \lesssim \frac{1}{\Delta t}\left\|\phi_{h, \Delta t}\right\|_{0, \frac{1}{2}, \Gamma, *}
$$

provided $\phi_{h, \Delta t} \in V_{h, \Delta t}^{p, q}$, the space of piecewise polynomials defined in (2.9).

Theorem 3.1. Let $\psi \in H_{\sigma}^{1}\left(\mathbb{R}^{+}, \widetilde{H}^{\frac{1}{2}}(\Gamma)\right)$ be the solution of (2.8), $\psi_{h, \Delta t} \in V_{h, \Delta t}^{p, q}$ the solution of (2.11). Then there holds:

$$
\begin{aligned}
& \left\|\psi-\psi_{h, \Delta t}\right\|_{0, \frac{1}{2}, \Gamma, *} \\
\lesssim & \left\|g_{h, \Delta t}-g\right\|_{1,-\frac{1}{2}, \Gamma}+\left(1+(\Delta t)^{-1}\right) \inf _{\phi_{h, \Delta t} \in V_{h, \Delta t}^{p, q}}\left\|\psi-\phi_{h, \Delta t}\right\|_{1, \frac{1}{2}, \Gamma, *} .
\end{aligned}
$$

Proof. We start with the coercivity estimate, Theorem 2.1b), applied to $\psi_{h, \Delta t}-\phi_{h, \Delta t} \in$ $H_{\sigma}^{1}\left(\mathbb{R}^{+}, H^{\frac{1}{2}}(\Gamma)\right)$, where $\phi_{h, \Delta t} \in V_{h, \Delta t}^{p, q}$ is arbitrary:

$$
\begin{aligned}
& \left\|\psi_{h, \Delta t}-\phi_{h, \Delta t}\right\|_{0, \frac{1}{2}, \Gamma, *}^{2} \\
\lesssim & \int_{\mathbb{R}^{+} \times \Gamma} e^{-2 \sigma t}\left(W\left(\psi_{h, \Delta t}-\phi_{h, \Delta t}\right)\right) \partial_{t}\left(\psi_{h, \Delta t}-\phi_{h, \Delta t}\right) d t d s_{x} \\
= & \int_{\mathbb{R}^{+} \times \Gamma} e^{-2 \sigma t}\left(W\left(\psi_{h, \Delta t}-\psi\right)\right) \partial_{t}\left(\psi_{h, \Delta t}-\phi_{h, \Delta t}\right) d t d s_{x} \\
& \quad+\int_{\mathbb{R}^{+} \times \Gamma} e^{-2 \sigma t}\left(W\left(\psi-\phi_{h, \Delta t}\right)\right) \partial_{t}\left(\psi_{h, \Delta t}-\phi_{h, \Delta t}\right) d t d s_{x} .
\end{aligned}
$$

In the second line we have added and subtracted the term with $\psi$. For the first term we obtain using the discretized weak form (2.11) and the continuity of the duality pairing:

$$
\begin{aligned}
& \int_{\mathbb{R}^{+} \times \Gamma} e^{-2 \sigma t}\left(W\left(\psi_{h, \Delta t}-\psi\right)\right) \partial_{t}\left(\psi_{h, \Delta t}-\phi_{h, \Delta t}\right) d t d s_{x} \\
= & \int_{\mathbb{R}^{+} \times \Gamma} e^{-2 \sigma t}\left(g_{h, \Delta t}-g\right) \partial_{t}\left(\psi_{h, \Delta t}-\phi_{h, \Delta t}\right) d t d s_{x} \\
\leq & \left\|g_{h, \Delta t}-g\right\|_{1,-\frac{1}{2}, \Gamma}\left\|\partial_{t}\left(\psi_{h, \Delta t}-\phi_{h, \Delta t}\right)\right\|_{-1, \frac{1}{2}, \Gamma, *} .
\end{aligned}
$$


For the second term the continuity of duality pairing and the mapping properties of $W$ in Theorem 2.1a) show:

$$
\begin{aligned}
& \int_{\mathbb{R}^{+} \times \Gamma} e^{-2 \sigma t}\left(W\left(\psi-\phi_{h, \Delta t}\right)\right) \partial_{t}\left(\psi_{h, \Delta t}-\phi_{h, \Delta t}\right) d t d s_{x} \\
\leq & \left\|W\left(\psi-\phi_{h, \Delta t}\right)\right\|_{0,-\frac{1}{2}, \Gamma}\left\|\partial_{t}\left(\psi_{h, \Delta t}-\phi_{h, \Delta t}\right)\right\|_{0, \frac{1}{2}, \Gamma, *} \\
\lesssim & \left\|\psi-\phi_{h, \Delta t}\right\|_{1, \frac{1}{2}, \Gamma, *}\left\|\psi_{h, \Delta t}-\phi_{h, \Delta t}\right\|_{1, \frac{1}{2}, \Gamma, *} .
\end{aligned}
$$

We use the inverse inequality (3.1) in the time variable to estimate second factor:

$$
\left\|\psi_{h, \Delta t}-\phi_{h, \Delta t}\right\|_{1, \frac{1}{2}, \Gamma, *} \lesssim \frac{1}{\Delta t}\left\|\psi_{h, \Delta t}-\phi_{h, \Delta t}\right\|_{0, \frac{1}{2}, \Gamma, *} .
$$

Therefore we obtain

$$
\left\|\psi_{h, \Delta t}-\phi_{h, \Delta t}\right\|_{0, \frac{1}{2}, \Gamma, *} \lesssim\left\|g_{h, \Delta t}-g\right\|_{1,-\frac{1}{2}, \Gamma}+(\Delta t)^{-1}\left\|\psi-\phi_{h, \Delta t}\right\|_{1, \frac{1}{2}, \Gamma, *} .
$$

With the triangle inequality, one concludes

$$
\begin{gathered}
\left\|\psi-\psi_{h, \Delta t}\right\|_{0, \frac{1}{2}, \Gamma, *} \leq\left\|\psi-\phi_{h, \Delta t}\right\|_{0, \frac{1}{2}, \Gamma, *}+\left\|\psi_{h, \Delta t}-\phi_{h, \Delta t}\right\|_{0, \frac{1}{2}, \Gamma, *} \\
\lesssim\left\|g_{h, \Delta t}-g\right\|_{1,-\frac{1}{2}, \Gamma}+\left\|\psi-\phi_{h, \Delta t}\right\|_{0, \frac{1}{2}, \Gamma, *}+(\Delta t)^{-1}\left\|\psi-\phi_{h, \Delta t}\right\|_{1, \frac{1}{2}, \Gamma, *} .
\end{gathered}
$$

The a priori estimate follows.

\subsection{An a posteriori error estimate}

In this section we derive a simple computable error estimate, which can be used to steer adaptive mesh refinements based on the four steps

$$
\text { SOLVE } \longrightarrow \text { ESTIMATE } \longrightarrow \text { MARK } \longrightarrow \text { REFINE }
$$

as shown for the single layer potential in $[8,10,11]$. Because in practical computations we set $\sigma=0$, we derive the estimate on finite time intervals $[0, T]$, but as in these sources also $\mathbb{R}^{+}$ could be considered. Also, for simplicity we assume $g=g_{h, \Delta t}$. The weak formulation on $[0, T]$ reads as: Find $\psi \in H_{0}^{1}\left([0, T], \widetilde{H}^{\frac{1}{2}}(\Gamma)\right)$ such that for all $\Psi \in H_{0}^{1}\left([0, T], \widetilde{H}^{\frac{1}{2}}(\Gamma)\right)$ there holds:

$$
\int_{[0, T] \times \Gamma}(W \psi) \partial_{t} \Psi d t d s_{x}=\int_{[0, T] \times \Gamma} g \partial_{t} \Psi d t d s_{x} .
$$

Its Galerkin discretization is given by: Find $\psi_{h, \Delta t} \in V_{h, \Delta t}^{p, q}$ such that for all test functions $\Psi_{h, \Delta t} \in V_{h, \Delta t}^{p, q}$ there holds:

$$
\int_{[0, T] \times \Gamma}\left(W \psi_{h, \Delta t}\right) \partial_{t} \Psi_{h, \Delta t} d t d s_{x}=\int_{[0, T] \times \Gamma} g \partial_{t} \Psi_{h, \Delta t} d t d s_{x} .
$$

Instead of the coercivity estimate in Theorem $2.1 \mathrm{~b}$, the analysis of the scheme may be directly based on considerations of the energy

$$
E(u, t)=\frac{1}{2} \int_{\Omega^{i} \cup \Omega^{e}}\left(\left(\partial_{t} u\right)^{2}+|\nabla u|^{2}\right) d x .
$$


Following Ha Duong [13], if $u$ satisfies the wave equation outside $\Gamma$, the representation formula and Green's identity may be used to express the energy at time $t$ in terms of the hypersingular operator and $\left.u\right|_{\Gamma_{+}}-\left.u\right|_{\Gamma_{-}}=\varphi$, where $\left.u\right|_{\Gamma_{ \pm}}$denote the upper, resp. lower, side of $\Gamma$ :

$$
E(u, t)=\int_{[0, t] \times \Gamma}(W \varphi) \dot{\varphi} d s_{x} d \tau .
$$

The time dependent version of the trace theorem for functions of finite energy [13],

$$
\left\|\left.u\right|_{\Gamma_{ \pm}}\right\|_{0, \frac{1}{2}, \Gamma, *}^{2} \lesssim_{T} \int_{0}^{T} E(u, t) d t
$$

therefore results in:

Proposition 3.1. For every $\varphi \in H_{0}^{1}\left([0, T], \widetilde{H}^{\frac{1}{2}}(\Gamma)\right)$ there holds:

$$
\|\varphi\|_{0, \frac{1}{2}, \Gamma, *}^{2} \lesssim_{T} \int_{0}^{T} \int_{[0, t] \times \Gamma}(W \varphi) \dot{\varphi} d s_{x} d \tau d t .
$$

We may now derive an a posteriori error estimate.

Theorem 3.2. Let $\psi \in H_{0}^{1}\left([0, T], \widetilde{H}^{\frac{1}{2}}(\Gamma)\right)$ be the solution of $(3.2), \psi_{h, \Delta t} \in V_{h, \Delta t}^{p, q}$ the solution of (3.3). Assume that

$$
R=g-W \psi_{h, \Delta t} \in H^{1}\left([0, T], \widetilde{H}^{-1 / 2}(\Gamma)\right) .
$$

Then there holds:

$$
\left\|\psi-\psi_{h, \Delta t}\right\|_{0, \frac{1}{2}, \Gamma, *} \lesssim\|R\|_{1,-\frac{1}{2}, \Gamma} \cdot
$$

Proof. From Proposition 3.1 we first note that

$$
\left\|\psi-\psi_{h, \Delta t}\right\|_{0, \frac{1}{2}, \Gamma, *}^{2} \lesssim_{T} \int_{0}^{T} \int_{0}^{t} \int_{\Gamma}\left(W\left(\psi-\psi_{h, \Delta t}\right)\right) \partial_{t}\left(\psi-\psi_{h, \Delta t}\right) d s_{x} d \tau d t .
$$

Using the continuous weak formulation (2.8), then its discretization (2.11), we have for all $\Psi_{h, \Delta t} \in V_{h, \Delta t}^{p, q}:$

$$
\begin{aligned}
& \int_{0}^{T} \int_{0}^{t} \int_{\Gamma}\left(W\left(\psi-\psi_{h, \Delta t}\right)\right) \partial_{t}\left(\psi-\psi_{h, \Delta t}\right) d s_{x} d \tau d t \\
= & \int_{0}^{T} \int_{0}^{t} \int_{\Gamma} g \partial_{t}\left(\psi-\psi_{h, \Delta t}\right) d s_{x} d \tau d t-\int_{0}^{T} \int_{0}^{t} \int_{\Gamma}\left(W \psi_{h, \Delta t}\right) \partial_{t}\left(\psi-\psi_{h, \Delta t}\right) d s_{x} d \tau d t \\
= & \int_{0}^{T} \int_{0}^{t} \int_{\Gamma} g \partial_{t}\left(\psi-\Psi_{h, \Delta t}\right) d s_{x} d \tau d t-\int_{0}^{T} \int_{0}^{t} \int_{\Gamma}\left(W \psi_{h, \Delta t}\right) \partial_{t}\left(\psi-\Psi_{h, \Delta t}\right) d s_{x} d \tau d t \\
= & \int_{0}^{T} \int_{0}^{t} \int_{\Gamma}\left(g-W \psi_{h, \Delta t}\right) \partial_{t}\left(\psi-\Psi_{h, \Delta t}\right) d s_{x} d \tau d t
\end{aligned}
$$

The last term may be estimated by interchanging the time integrals and duality:

$$
\begin{aligned}
& \int_{0}^{T} \int_{0}^{t} \int_{\Gamma}\left(g-W \psi_{h, \Delta t}\right) \partial_{t}\left(\psi-\Psi_{h, \Delta t}\right) d s_{x} d \tau d t \\
= & \int_{0}^{T}(T-t) \int_{\Gamma}\left(g-W \psi_{h, \Delta t}\right) \partial_{t}\left(\psi-\Psi_{h, \Delta t}\right) d s_{x} d t \\
\leq & T\|R\|_{1,-\frac{1}{2}, \Gamma}\left\|\psi-\Psi_{h, \Delta t}\right\|_{0, \frac{1}{2}, \Gamma, *} .
\end{aligned}
$$

We use $\Psi_{h, \Delta t}=\psi_{h, \Delta t}$ to obtain the estimate. 


\section{Algorithmic Considerations}

\subsection{Implementation of $W$}

We set $\tau=t-|x-y|$ and use $\sigma=0$. Citing a formula for $W$ from Ha-Duong [12], Lemma 4b), we have:

$$
\begin{aligned}
& \int_{\mathbb{R}^{+} \times \Gamma}(W \psi) \partial_{t} \Psi d t d s_{x} \\
= & \frac{1}{2 \pi} \int_{0}^{\infty} \int_{\Gamma \times \Gamma}\left\{\frac{-n_{x} \cdot n_{y}}{|x-y|} \dot{\psi}(\tau, y) \ddot{\Psi}(t, x)+\frac{\left(\operatorname{curl}_{\Gamma} \psi\right)(\tau, y) \cdot\left(\operatorname{curl}_{\Gamma} \dot{\Psi}\right)(t, x)}{|x-y|}\right\} d s_{y} d s_{x} d t .
\end{aligned}
$$

We use piecewise linear ansatz functions $\varphi_{i}^{1}(x) \beta^{m, 1}(t)$ from the space $V_{h, \Delta t}^{1,1}($ see $(2.9))$ in space and time:

$$
\psi_{h, \Delta t}(t, x)=\sum_{m=1}^{N_{t}} \sum_{i=1}^{N_{s}} c_{i}^{m} \varphi_{i}^{1}(x) \beta^{m, 1}(t),
$$

where $\beta^{m, 1}(t)=(\Delta t)^{-1}\left(\left(t-t_{m-1}\right) \gamma^{m}(t)-\left(t-t_{m+1}\right) \gamma^{m+1}(t)\right)$ and $\gamma^{j}(t)=\beta^{j, 0}$ is the characteristic function of $\left(t_{j-1}, t_{j}\right]$. For algorithmic reasons, to obtain the time-stepping scheme below, we choose test functions $\dot{\Psi}_{h, \Delta t}(t, x)=\varphi_{j}^{1}(x) \gamma^{n}(t)$, which are piecewise constant in time and piecewise linear in space. Expanding (4.1) for ansatz functions $\psi_{h, \Delta t}$ of the form (4.2) results in:

$$
\int_{\mathbb{R}^{+} \times \Gamma} W \psi_{h, \Delta t}(t, x) \partial_{t} \Psi_{h, \Delta t}(t, x) d t d s_{x}=A-B
$$

with

$$
\begin{aligned}
A & =\sum_{m=1}^{N_{t}} \sum_{i=1}^{N_{s}} \frac{c_{i}^{m}}{2 \pi} \int_{\Gamma \times \Gamma} \frac{1}{|x-y|} \int_{0}^{\infty} \beta^{m, 1}(\tau) \operatorname{curl}_{\Gamma} \varphi_{i}^{1}(y) \gamma^{n}(t) \operatorname{curl}_{\Gamma} \varphi_{j}^{1}(x) d t d s_{y} d s_{x} \\
B & =\frac{1}{2 \pi} \int_{0}^{\infty} \int_{\Gamma \times \Gamma} \frac{n_{x} \cdot n_{y}}{|x-y|}\left(\sum_{m=1}^{N_{t}} \sum_{i=1}^{N_{s}} c_{i}^{m} \dot{\beta}^{m, 1}(\tau) \varphi_{i}^{1}(y)\right) \dot{\gamma}^{n}(t) \varphi_{j}^{1}(x) d s_{y} d s_{x} d t \\
& =\sum_{m=1}^{N_{t}} \sum_{i=1}^{N_{s}} \frac{c_{i}^{m}}{2 \pi} \int_{\Gamma \times \Gamma} \frac{n_{x} \cdot n_{y}}{|x-y|} \varphi_{i}^{1}(y) \varphi_{j}^{1}(x)\left(\int_{0}^{\infty} \dot{\beta}^{m, 1}(\tau) \dot{\gamma}^{n}(t) d t\right) d s_{y} d s_{x} .
\end{aligned}
$$

Using, in particular, that the derivative $\dot{\gamma}^{n}=\delta_{t_{n-1}}-\delta_{t_{n}}$ is a difference of Dirac distributions, we first compute

$$
\begin{aligned}
& \int_{0}^{\infty} \dot{\beta}^{m, 1}(\tau) \dot{\gamma}^{n}(t) d t \\
= & (\Delta t)^{-1}\left(2\left(H\left(t_{n-m}-|x-y|\right)-H\left(t_{n-m-1}-|x-y|\right)\right)-H\left(t_{n-m+1}-|x-y|\right)\right. \\
& \left.\quad+H\left(t_{n-m}-|x-y|\right)-H\left(t_{n-m-1}-|x-y|\right)+H\left(t_{n-m-2}-|x-y|\right)\right) \\
& \quad-(\Delta t)^{-1}\left(\chi_{E_{n-m}}(x, y)-2 \chi_{E_{n-m-1}}(x, y)+\chi_{E_{n-m-2}}\right) .
\end{aligned}
$$

Here, for $l \in \mathbb{N}_{0}$ we define the light cone $E_{l}=\left\{(x, y) \in \Gamma \times \Gamma: t_{l} \leq|x-y| \leq t_{l+1}\right\} \subset \Gamma \times \Gamma$, and $\chi_{E_{l}}(x, y)=1$ if $(x, y) \in E_{l}$, and $=0$ otherwise. The second equality is verified by calculating 
both sides for $(x, y) \in E_{l}$. To conclude:

$$
\begin{aligned}
& B=\sum_{m=1}^{N_{t}} \sum_{i=1}^{N_{s}} c_{i}^{m}\left[-\int_{E_{n-m}} \frac{\left(n_{x} \cdot n_{y}\right)(\Delta t)^{-1} \varphi_{i}^{1}(y) \varphi_{j}^{1}(x)}{2 \pi|x-y|} d s_{y} d s_{x}\right. \\
& \left.\quad+2 \int_{E_{n-m-1}} \frac{\left(n_{x} \cdot n_{y}\right)(\Delta t)^{-1} \varphi_{i}^{1}(y) \varphi_{j}^{1}(x)}{2 \pi|x-y|} d s_{y} d s_{x}-\int_{E_{n-m-2}} \frac{\left(n_{x} \cdot n_{y}\right)(\Delta t)^{-1} \varphi_{i}^{1}(y) \varphi_{j}^{1}(x)}{2 \pi|x-y|} d s_{y} d s_{x}\right] .
\end{aligned}
$$

We now consider $A$ :

$$
\begin{aligned}
A & =\sum_{m=1}^{N_{t}} \sum_{i=1}^{N_{s}} \frac{c_{i}^{m}}{2 \pi} \int_{\Gamma \times \Gamma} \frac{1}{|x-y|} \int_{0}^{\infty} \beta^{m, 1}(\tau) \operatorname{curl}_{\Gamma} \varphi_{i}^{1}(y) \gamma^{n}(t) \operatorname{curl}_{\Gamma} \varphi_{j}^{1}(x) d t d s_{y} d s_{x} \\
& =\sum_{m=1}^{N_{t}} \sum_{i=1}^{N_{s}} \frac{c_{i}^{m}}{2 \pi} \int_{\Gamma \times \Gamma} \frac{1}{|x-y|} \operatorname{curl}_{\Gamma} \varphi_{i}^{1}(y) \operatorname{curl}_{\Gamma} \varphi_{j}^{1}(x) \int_{0}^{\infty} \beta^{m, 1}(\tau) \gamma^{n}(t) d t d s_{y} d s_{x} .
\end{aligned}
$$

An explicit calculation of the integral shows

$$
\begin{aligned}
& \int_{0}^{\infty} \beta^{m, 1}(\tau) \gamma^{n}(t) d t=\int_{0}^{\infty}(\Delta t)^{-1}\left(\left(t-|x-y|-t_{m}\right) \gamma^{m}(t-|x-y|)\right. \\
& \left.\quad-\left(t-|x-y|-t_{m+1}\right) \gamma^{m+1}(t-|x-y|)\right) \gamma^{n}(t) d t \\
= & (\Delta t)^{-1} \int_{0}^{\infty}\left(t-|x-y|-t_{m}\right) \gamma^{m}(t-|x-y|) \gamma^{n}(t) d t \\
& \quad-(\Delta t)^{-1} \int_{0}^{\infty}\left(t-|x-y|-t_{m+1}\right) \gamma^{m+1}(t-|x-y|) \gamma^{n}(t) d t \\
=(2 \Delta t)^{-1}\left(|x-y|^{2}-2|x-y| t_{n-m+1}+t_{n-m+1}^{2}\right) \chi_{E_{n-m}}(x, y) & \quad+(2 \Delta t)^{-1}\left(|x-y|^{2}-2|x-y| t_{n-m-2}+t_{n-m-2}^{2}\right) \chi_{E_{n-m-2}}(x, y) \\
& \quad+(2 \Delta t)^{-1}\left(-2|x-y|^{2}+2|x-y|\left(t_{n-m}+t_{n-m-1}\right)-\left(t_{n-m}^{2}+t_{n-m-1}^{2}\right)+2(\Delta t)^{2}\right) \chi_{E_{n-m-1}}(x, y) \\
=: & \Upsilon^{n-m}(x, y) .
\end{aligned}
$$

Here we use the definition of $E_{l}$ from above. Therefore

$$
A=\sum_{m=1}^{N_{t}} \sum_{i=1}^{N_{s}} \frac{c_{i}^{m}}{2 \pi} \int_{\Gamma \times \Gamma} \frac{1}{|x-y|} \operatorname{curl}_{\Gamma} \varphi_{i}^{1}(y) \operatorname{curl}_{\Gamma} \varphi_{j}^{1}(x) \Upsilon^{n-m}(x, y) d s_{y} d s_{x} .
$$

\subsection{Marching-on-in-time scheme}

In terms of the coefficients $c_{i}^{m}$ with respect to the basis functions we note from the formulas for $A$ and $B$ in Section 4.1 that

$$
\int_{\mathbb{R}^{+} \times \Gamma} W \psi_{h, \Delta t}(t, x) \partial_{t} \Psi_{h, \Delta t}(t, x) d t d s_{x}=\sum_{m=1}^{N_{t}} W^{n-m} c^{m} .
$$

Here $W^{n-m}$ is a matrix which has $A-B$ as entries.

Similarly we have for the right hand side

$$
g_{h, \Delta t}(t, x)=\sum_{m=1}^{N_{t}} \sum_{i=1}^{N_{s}} g_{i}^{m} \beta^{m, 1}(t) \varphi_{i}^{1}(x)
$$


In matrix-vector notation we obtain using the stiffness matrix $I_{i, j}=\int_{\Gamma} \xi_{i}(x) \xi_{j}(x) d s_{x}$ :

$$
\sum_{m=1}^{N_{t}} W^{n-m} c^{m}=\frac{\Delta t}{2} I\left(g^{n-1}+g^{n}\right)
$$

i.e., an explicit time stepping scheme, known as the marching-on-in-time (MOT) algorithm:

$$
W^{0} c^{n}=\frac{\Delta t}{2} I\left(g^{n-1}+g^{n}\right)-\sum_{m=1}^{n-1} W^{n-m} c^{m} .
$$

\subsection{Leading contribution of absorbing boundary conditions to $K^{\prime}$}

To consider the leading contribution of an absorbing half-space, we show that the leading part of the new term $\Sigma$ in the fundamental solution (2.1a) for the absorbing half-space can be implemented as a minor modification of the pure Neumann problem, $\alpha=\Sigma=0$. For this, let $y^{\prime}=\left(y_{1}, y_{2},-y_{3}\right)$ the reflection of $y$ on the $y_{3}=0$-plane, $\vartheta=x_{3}+y_{3}$ and $R^{2}=$ $\left(x_{1}-y_{1}\right)^{2}+\left(x_{2}-y_{2}\right)^{2}$. We compute that the contribution of $\Sigma$ to the operator $K^{\prime}$ as in (2.4),

$$
\left\langle K_{3}^{\prime} \varphi, \Psi\right\rangle:=2 \int_{\mathbb{R}^{+} \times \Gamma} \int_{\mathbb{R}^{+} \times \Gamma} \frac{\partial \Sigma}{\partial n_{x}}(t-\tau, x, y) \varphi(\tau, y) \Psi(t, x) d \tau d s_{y} d t d s_{x},
$$

is given by

$$
\frac{\alpha}{\pi} \int_{\mathbb{R}^{+} \times \Gamma} \int_{\mathbb{R}^{+} \times \Gamma} \frac{\partial}{\partial n_{x}}\left(\frac{\partial}{\partial \tau}\left[\frac{H\left(t-\tau-\left|x-y^{\prime}\right|\right)}{\sqrt{\left(t-\tau+\alpha \vartheta_{3}\right)^{2}+\left(\alpha^{2}-1\right) R^{2}}}\right] \varphi(\tau, y)\right) \Psi(t, x) d s_{y} d \tau d s_{x} d t .
$$

If we define

$$
A(t, \tau):=\sqrt{\left(t-\tau+\alpha \vartheta_{3}\right)^{2}+\left(\alpha^{2}-1\right) R^{2}},
$$

an integration by parts in $\tau$ shows that

$$
\left\langle K_{3}^{\prime} \varphi, \Psi\right\rangle=-\frac{\alpha}{\pi} \int_{\mathbb{R}^{+} \times \Gamma \mathbb{R}^{+} \times \Gamma} \int_{\frac{\partial}{\partial n_{x}}}\left(\left[\frac{H\left(t-\tau-\left|x-y^{\prime}\right|\right)}{A(t, \tau)}\right] \dot{\varphi}(\tau, y)\right) \Psi(t, x) d s_{y} d s_{x} d \tau d t .
$$

In a physically motivated approximation, we neglect the $x$-derivative of $A$ :

$$
\frac{\partial}{\partial n_{x}}\left[\frac{H\left(t-\tau-\left|x-y^{\prime}\right|\right)}{A(t, \tau)}\right]=-\frac{\vec{n}_{x} \cdot\left(x-y^{\prime}\right)}{\left|x-y^{\prime}\right| A(t, \tau)} \delta\left(t-\tau-\left|x-y^{\prime}\right|\right)+\cdots
$$

With piecewise constant ansatz and test functions in space and time, we obtain

$$
\begin{aligned}
\left\langle K_{3}^{\prime} \varphi_{h, \Delta t}, \Psi_{h, \Delta t}\right\rangle \simeq \frac{\alpha}{\pi} \int_{\Gamma \times \Gamma} \varphi_{i}^{0}(x) \varphi_{j}^{0}(y)\left(\gamma^{m}\left(t_{n-1}+\left|x-y^{\prime}\right|\right)-\gamma^{m}\left(t_{n}+\left|x-y^{\prime}\right|\right)\right) \times \\
\\
\quad \cdots \times \frac{n_{x} \cdot\left(x-y^{\prime}\right)}{\left|x-y^{\prime}\right| A\left(t_{n-1}+\left|x-y^{\prime}\right|, t_{n-1}\right)} d s_{x} d s_{y} .
\end{aligned}
$$

This term is easily included in the contributions of the first two terms of the fundamental solution, see [2]. 


\section{Numerical Results}

\subsection{Neumann problem exterior to the sphere}

In the following, we present numerical results for the Neumann problem (1.1), using the time domain boundary element formulations of the first, resp. second kind, (2.8) and (2.7).

In the special case where $\Gamma=S^{2}=\left\{x \in \mathbb{R}^{3}:|x|=1\right\}$ is the unit sphere, for simple right hand sides exact solutions for the densities may be found in [20]. We recall that for the hypersingular equation $W \psi=g$ with $g(t, x)=g(t)$, the solution has the following form [20]:

$$
\begin{aligned}
\psi(t, x)=\psi(t) & =-\int_{0}^{t} g(t-\tau) \cosh (\tau) d \tau \\
+ & \sum_{k=1}^{\lfloor t / 2\rfloor} \sum_{l=1}^{k}(-1)^{k+1} \int_{2 k}^{t} c_{k, l}(\tau-2 k)^{k-l+1} e^{\tau-2 k} \dot{g}(t-\tau) d \tau
\end{aligned}
$$

Here

$$
c_{k, l}:=\left(\begin{array}{c}
k-1 \\
l-1
\end{array}\right) \frac{2^{k-l}}{(k-l+1) !} .
$$

For the corresponding equation of the second kind, $\left(-I d+K^{\prime}\right) \varphi=g$, again with $g(t, x)=g(t)$, the exact solution is given by

$$
\varphi(t, x)=-\sum_{k=0}^{\lfloor t / 2\rfloor} g(t-2 k)+\sum_{k=0}^{\lfloor t / 2\rfloor} \int_{2 k}^{t} e^{-(\tau-2 k)} g(t-\tau) d \tau
$$

As $\varphi$ is independent of $x$, the $L^{2}(\Gamma)$ norm turns out to be $\|\varphi(t)\|_{L^{2}(\Gamma)}=2 \sqrt{\pi}|\varphi(t)|$, and similarly for $\psi$.

Example 1. In the first numerical experiment, we look for solutions to $W \psi=g$, resp. $(-I d+$ $\left.K^{\prime}\right) \varphi=g$ with $g(t, x)=g(t)=\sin \left(\frac{t^{2}}{8}\right) \cos \left(t^{2}\right)$ on $\Gamma=S^{2}$ for the time interval [0, 12]. We use the time domain boundary element formulations (2.8) and (2.7) and compare the numerical solutions with the exact solutions from (5.1), resp. (5.2). For the discretization, we use the discretized tensor product spaces $V_{h, \Delta t}^{p, q}$ from (2.9) and follow Section 4. In particular, we use piecewise linear ansatz functions $V_{h, \Delta t}^{1,1}$ for (2.8), resp. piecewise constant ansatz functions $V_{h, \Delta t}^{0,0}$ for (2.7). The choice of test functions allows us to solve the discretised space time equations using the marching-on-in-time scheme from Section 4.2. To approximate the sphere, we start from a regular icosahedron with 20 faces. In each refinement step, we divide both the time step $\Delta t$ and mesh size $h$ by 2 and project the new nodes back onto $S^{2}$. The ratio $\Delta t / h \approx 0.6$ remains approximately constant.

Figure 5.1 depicts $\left\|\psi_{h, \Delta t}\right\|_{L^{2}\left(\Gamma_{h}\right)}$ for the numerical solution as a function of $t$ for meshes with 320, 1280, 5120, resp. 20480 triangles and compares it to $\|\psi(t)\|_{L^{2}(\Gamma)}$. In Figure 5.2 we show that the absolute value of the difference $\left\|\psi_{h, \Delta t}\right\|_{L^{2}\left(\Gamma_{h}\right)}-\|\psi\|_{L^{2}(\Gamma)}$ remains uniformly bounded as a function of time. When the number of degrees of freedom is increased, this error tends to 0 uniformly over the whole time interval, as is expected for a space-time Galerkin method.

For comparison, in Figure 5.3 we plot $\left\|\varphi_{h, \Delta t}\right\|_{L^{2}\left(\Gamma_{h}\right)}$ for the numerical solution of the discretized equation of the second kind. Figure 5.6 compares the $L^{2}([0, T] \times \Gamma)$-norm of the error for the resulting densities $\varphi$ (pink) resp. $\psi$ (blue) vs. the number of degrees of freedom, i.e. the number of time steps times spatial degrees of freedom. The rate of convergence for the Galerkin 


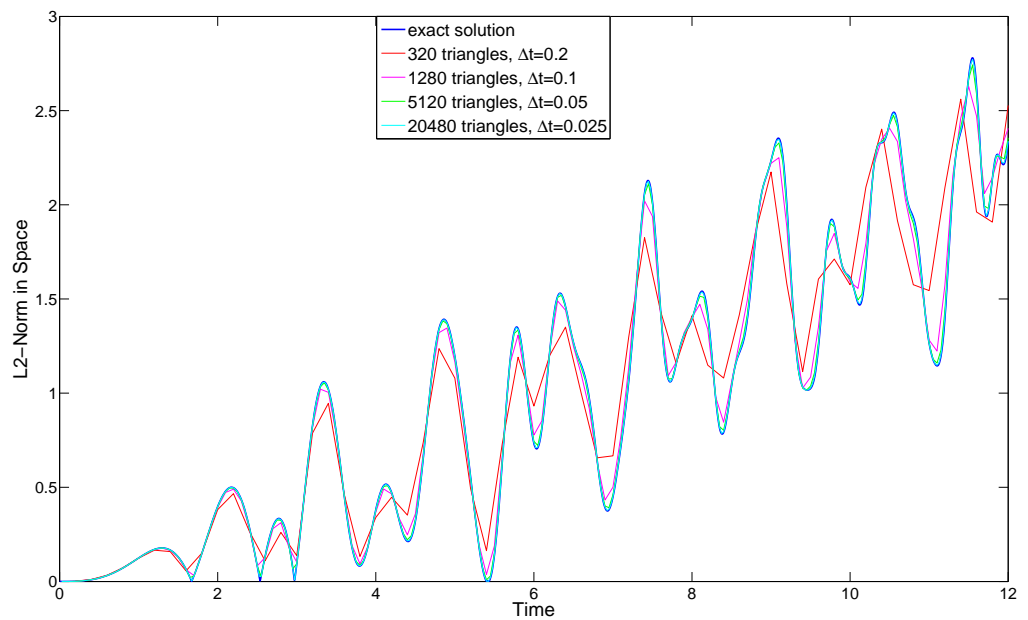

Fig. 5.1.: $L^{2}\left(\Gamma_{h}\right)$-norm of the solution to the hypersingular equation (2.8) for Example 1.

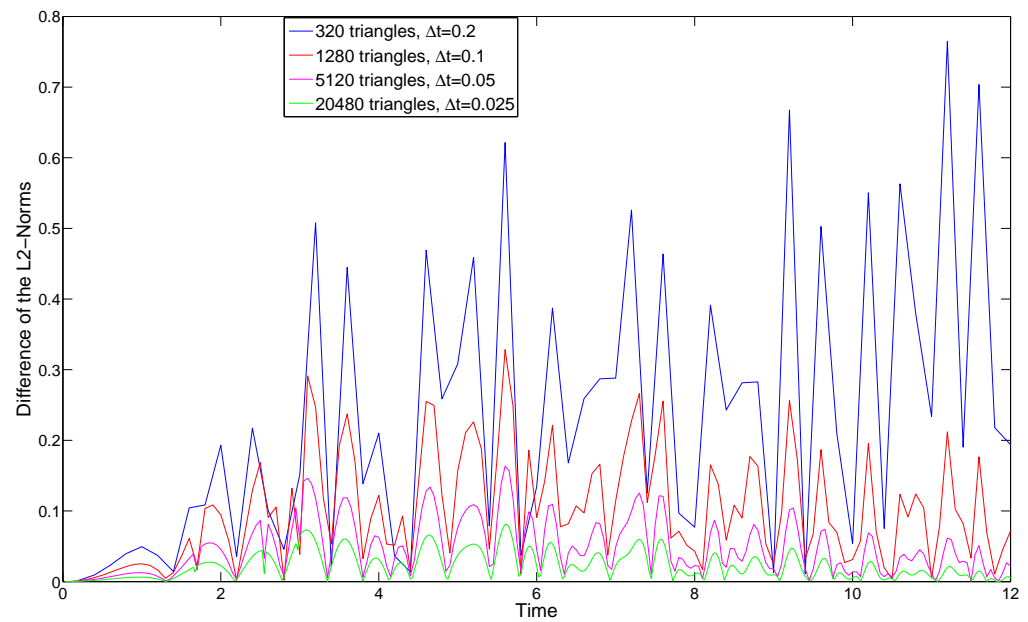

Fig. 5.2.: Absolute error $\left\|\psi_{h, \Delta t}\right\|_{L^{2}\left(\Gamma_{h}\right)}-\|\psi\|_{L^{2}(\Gamma)}$ as a function of time for the hypersingular equation (2.8), Example 1.

solutions of the hypersingular equation is approximately 1.0 in terms of degrees of freedom, or 3.0 in terms of $h$, compared to a rate 0.65 in degrees of freedom, 1.96 in $h$, for the Galerkin solutions of the integral equation of the second kind. Even for the coarsest discretization with 320 triangles and 60 time steps (19200 DOF for (2.7), 9720 DOF for (2.8)) the Galerkin error in $L^{2}([0, T] \times \Gamma)$ for the density $\psi$ is significantly lower for the hypersingular equation (2.8).

Example 2. We complement Example 1 with a second experiment in the same geometry, where $g(t, x)=t^{4} e^{-2 t}$. In this case, the exact solution $\psi$ to the hypersingular equation is approximately linear in the time interval [2,12], see Figure 5.4. Figure 5.6 shows a correspondingly higher rate of convergence 1.6 in degrees of freedom, 4.8 in $h$ (light blue curve), down to $L^{2}$-errors of $10^{-8}$. Even though the solution to the equation of the second kind (Figure 5.5) 
is far from linear, the rate of convergence 1.34 in degrees of freedom, 4.1 in $h$, from Figure 5.6 similarly indicates higher regularity of the solution compared to Example 1.

In both examples, the rates of convergence go beyond what our a priori estimates from Section 3 would indicate even for discretizations with higher polynomial degrees, for a general geometry.

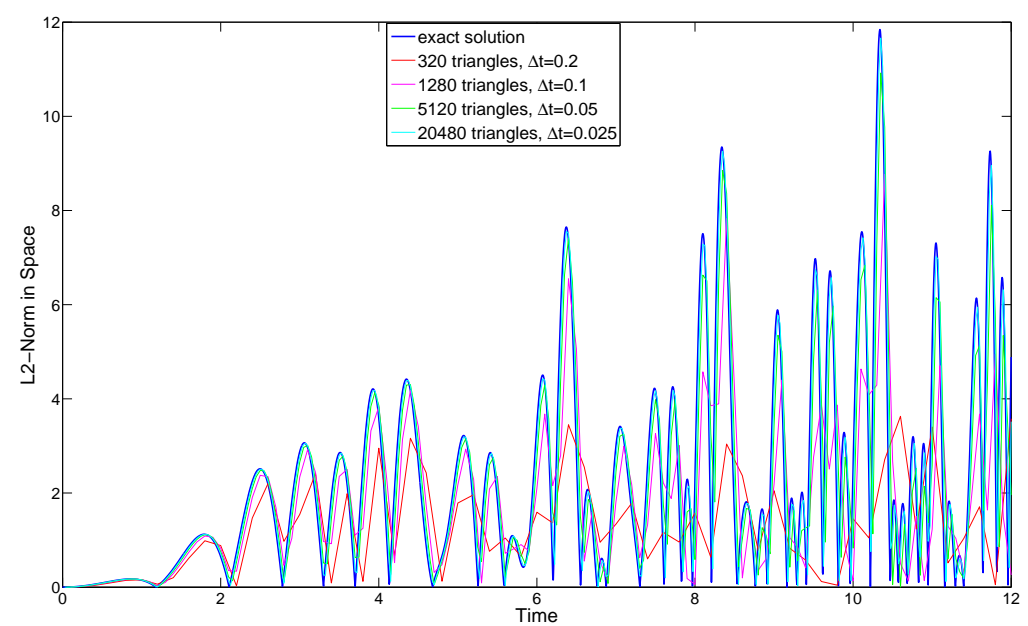

Fig. 5.3.: $L^{2}\left(\Gamma_{h}\right)$-norm of the solution to the integral equation (2.7) for Example 1.

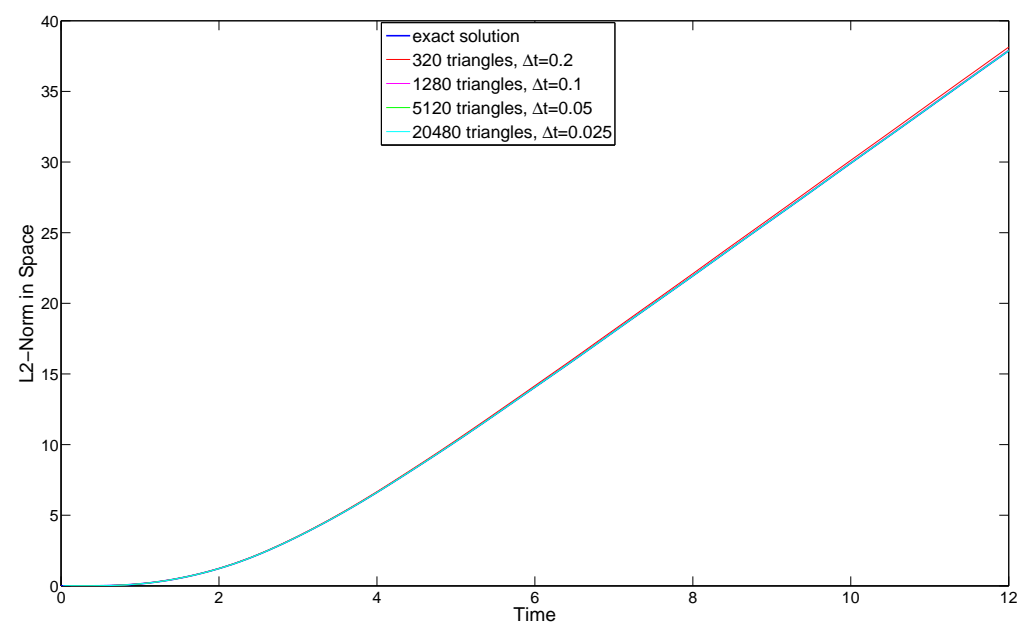

Fig. 5.4.: $L^{2}\left(\Gamma_{h}\right)$-norm of the solution to the hypersingular equation (2.8) for Example 2.

\subsection{Acoustic boundary conditions in a half-space}

In a further numerical experiment, we include the leading contribution of an acoustic halfspace $\mathbb{R}_{+}^{3}$ in our computations. The additional complications of the singular horn geometry between the emitter $\Gamma$ and $\mathbb{R}^{2} \times\{0\}$ are crucial for applications in traffic noise, and there is particular interest in properly modeling the reflectivity $\alpha$ of the street [2]. The Neumann and 


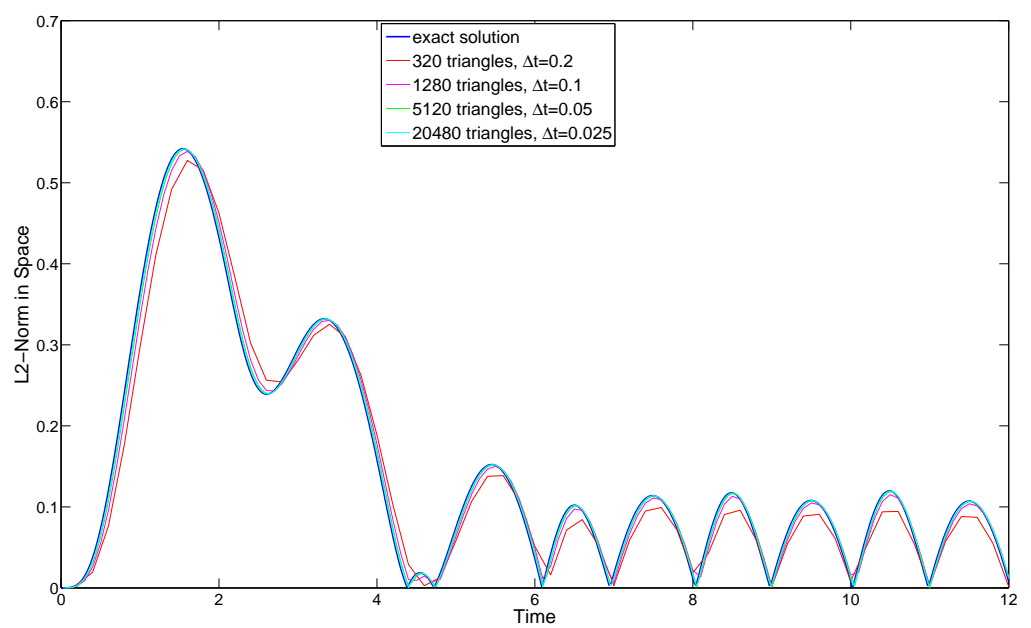

Fig. 5.5.: $L^{2}\left(\Gamma_{h}\right)$-norm of the solution to the integral equation (2.7) for Example 2.

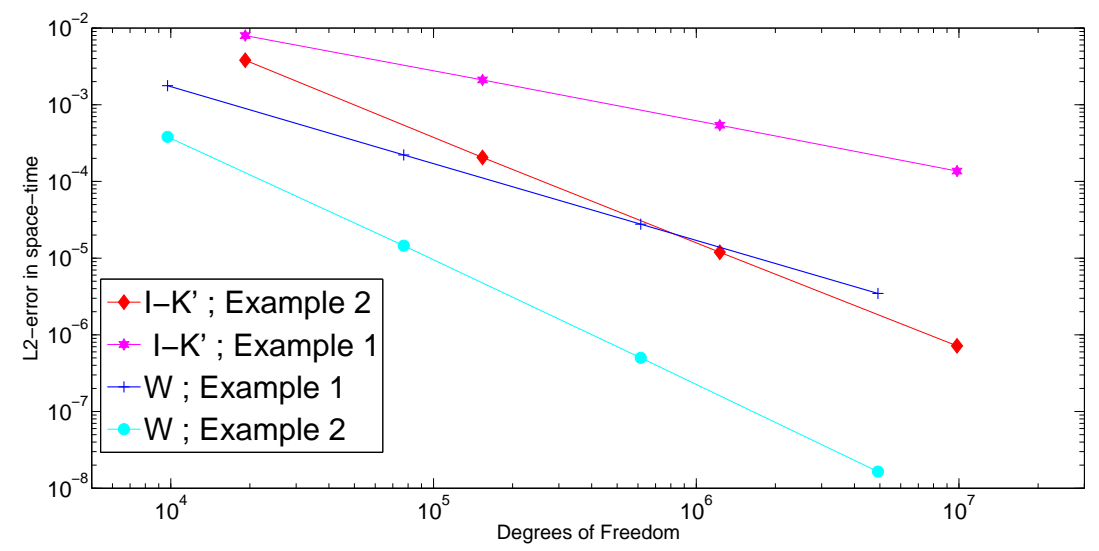

Fig. 5.6.: $L^{2}([0, T] \times \Gamma)$ error vs. degrees of freedom of the space-time mesh in Examples 1 and 2 , for the integral equations (2.7) resp. (2.8).

Dirichlet problems correspond to a reflectivity of $\alpha=0$ resp. $\alpha=\infty$, or physically hard vs. soft scattering.

Example 3. Again we consider the model geometry of the unit sphere, but now centered in $(0,0,1.63)$ in the half space $\mathbb{R}_{+}^{3}$ with acoustic boundary conditions

$$
\frac{\partial u}{\partial n}-\alpha \frac{\partial u}{\partial t}=0
$$

on $\mathbb{R}^{2} \times\{0\}$. We implement the Green's function corresponding to these boundary conditions in the half-space with an approximate third term, as described in Section 4.3. On $\Gamma$ Neumann conditions are imposed, $\frac{\partial u}{\partial n}=\frac{1}{2} g$. 


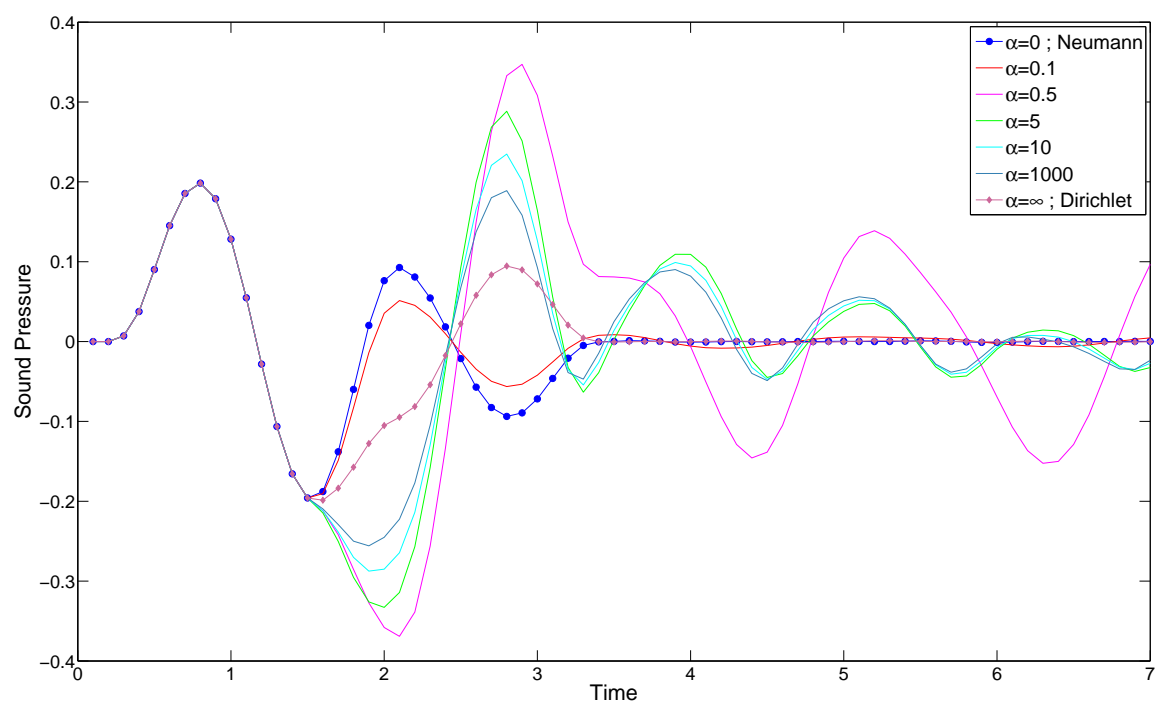

Fig. 5.7.: Sound pressure at $\left(\frac{1}{\sqrt{2}}, 0, \frac{1}{\sqrt{2}}\right)$ in $\mathbb{R}_{+}^{3}$ as a function of the reflectivity $\alpha$.

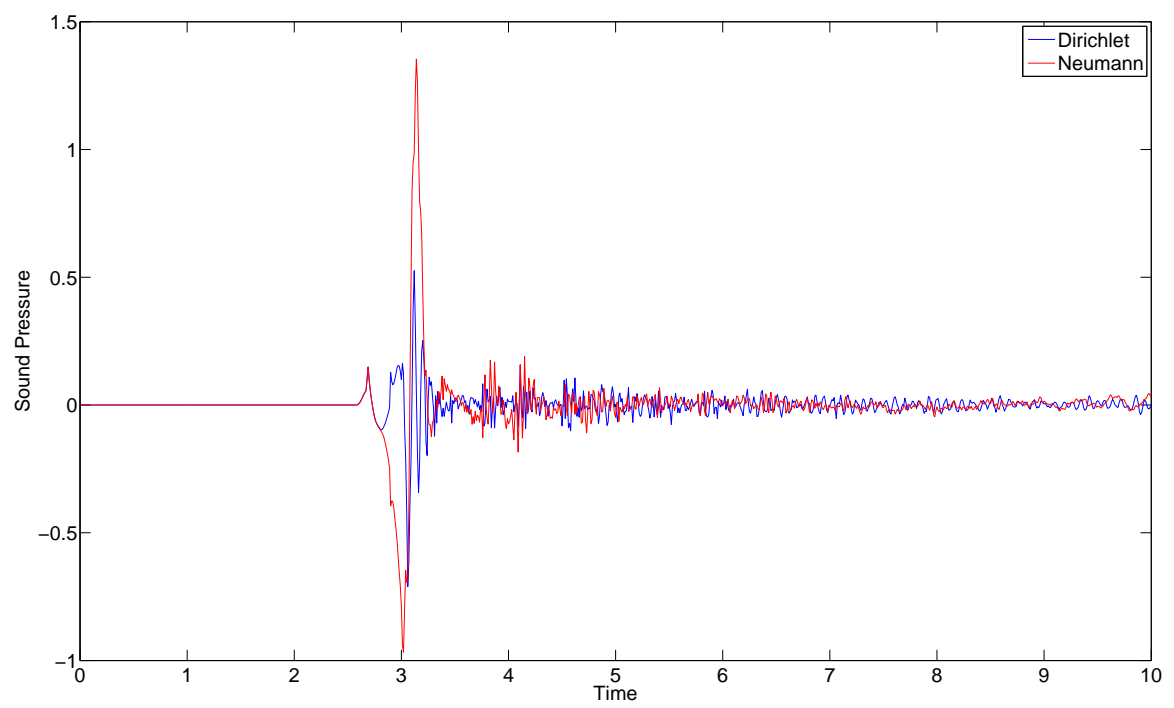

Fig. 5.8.: Sound pressure at $(2.8 \mathrm{~m}, 0,1.0 \mathrm{~m})$ as emitted by a car tire, Dirichlet or Neumann boundary conditions on the street.

We use the exact solution

$$
\begin{aligned}
u(t, x)=\frac{r_{+}-t}{2 r_{+}}\left[1+\cos \left(\frac{\pi\left(r_{+}-t\right)}{R}\right)\right] H\left(R-\left|r_{+}-t\right|\right) \\
+\frac{r_{-}-t}{2 r_{-}}\left[1+\cos \left(\frac{\pi\left(r_{-}-t\right)}{R}\right)\right] H\left(R-\left|r_{-}-t\right|\right)
\end{aligned}
$$




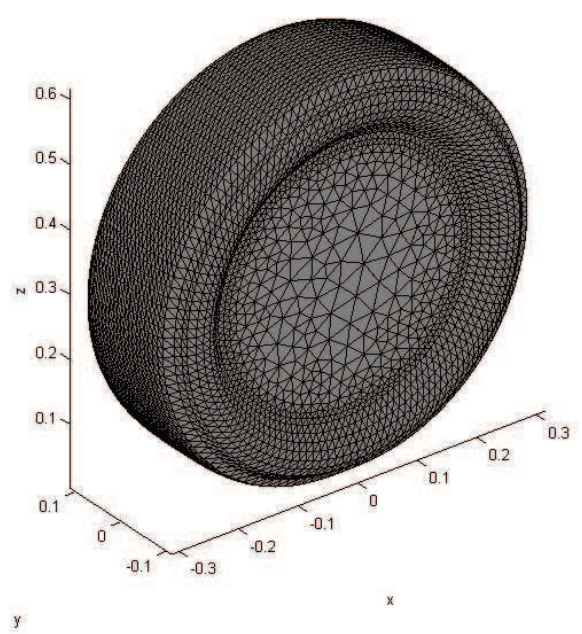

Fig. 5.9.: Mesh of the passenger car tire, Example 4.

of the Neumann problem with $\alpha=0$ to prescribe $g$ on $\Gamma$ :

$$
\begin{gathered}
\frac{1}{2} g=\left[\frac{t}{2 r_{+}^{2}}\left(1+\cos \left(\frac{\pi\left(r_{+}-t\right)}{R}\right)\right)-\frac{\pi}{R} \frac{r_{+}-t}{2 r_{+}} \sin \left(\frac{\pi\left(r_{+}-t\right)}{R}\right)\right] H\left(R-\left|r_{+}-t\right|\right) \\
+\frac{x^{2}+y^{2}+z^{2}-1.63^{2}}{r_{+} r_{-}}\left(\left[\frac{t}{2 r_{-}^{2}}\left(1+\cos \left(\frac{\pi\left(r_{-}-t\right)}{R}\right)\right)\right.\right. \\
\left.\left.-\frac{\pi}{R} \frac{r_{-}-t}{2 r_{-}} \sin \left(\frac{\pi\left(r_{-}-t\right)}{R}\right)\right] H\left(R-\left|r_{-}-t\right|\right)\right) .
\end{gathered}
$$

Here, $H(t)$ denotes the Heaviside function, $r_{+}=\left\|x_{1}, x_{2}, x_{3}-1.63\right\|$ and $r_{-}=\left\|x_{1}, x_{2}, x_{3}+1.63\right\|$ and $R=0.9$. While for Neumann boundary conditions $(\alpha=0)$ a single pulse is emitted from $\Gamma$ and reflected on $\mathbb{R}^{2} \times\{0\}$, the exact solution is not known for acoustic boundary conditions with reflectivity $\alpha \in(0, \infty)$ or Dirichlet boundary conditions, $\alpha=\infty$.

This acoustic problem is solved using the integral equation (2.7) of the second kind, where $K^{\prime}$ is defined from the modified Green's function as in Section 4.3. We use tensor products $V_{h, \Delta t}^{0,0}$ of piecewise constant ansatz and test functions in space and time on a fixed uniform mesh of 1280 triangles and $\Delta t=0.1$. Figure 5.7 shows the sound pressure $u_{h, \Delta t}(t, x)$ in the point $x=\left(\frac{1}{\sqrt{2}}, 0, \frac{1}{\sqrt{2}}\right)$ as a function of $t$ for different values of the coefficient $\alpha$. We note that the solution is independent of the boundary condition until the first reflected wave arrives in the point $x$. Increasing $\alpha$ from the Neumann problem $\alpha=0$ (blue) via $\alpha=0.1,0.5,5,10,1000$ to the Dirichlet problem $\alpha=+\infty$ (brown), we obtain a family of solutions which interpolates monotonously between these boundary conditions.

Depending on the reflectivity, we observe strong interference between the direct and reflected waves. Similar effects due to the singular horn geometry between the emitter and $\mathbb{R}^{2} \times\{0\}$ are observed in the sound emission of tires [2].

In the case of traffic noise, the resulting dependence on the reflectivity of the street will be crucial to take into account. This application is the content of our final example.

Example 4: For a problem in traffic noise, we illustrate the influence of the boundary conditions on the solution for the extreme cases of Dirichlet and Neumann conditions on the street. In this case $\Gamma$ is given by the mesh in Figure 5.9 with 6027 nodes of a grown slick 205/55R16 passenger car tyre, of diameter around $60 \mathrm{~cm}$, at 2 bar pressure and subject to $3415 \mathrm{~N}$ axle load 


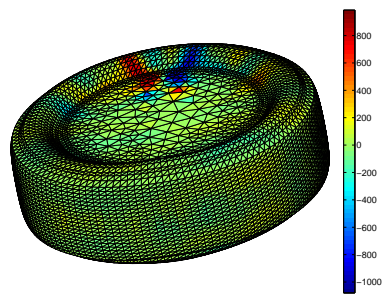

(a)

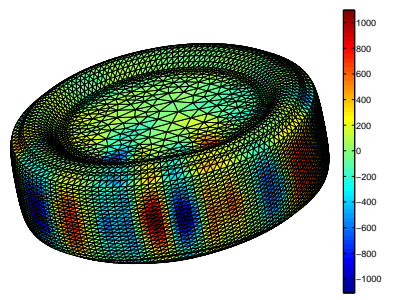

(b)

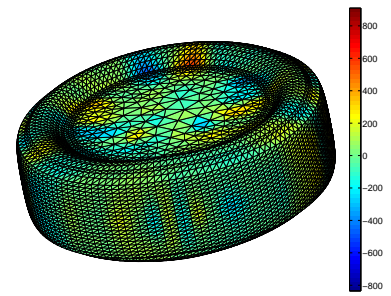

(c)

Fig. 5.10.: Visualization of the density for $\Delta t=0.01$, time step: 100 (a), 200 (b), 300 (c).

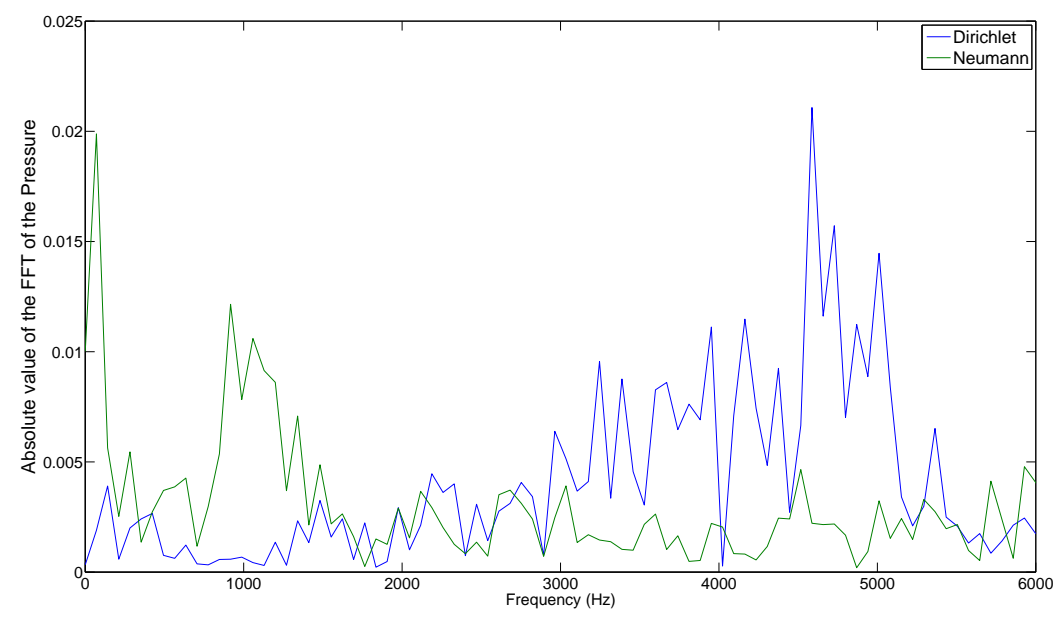

Fig. 5.11.: Sound pressure at $(2.8 \mathrm{~m}, 0,1.0 \mathrm{~m})$ in frequency domain, as emitted by a car tire.

at $50 \mathrm{~km} / \mathrm{h}$ on a street with an ISO 10844 surface [6]. The right hand side $g$ is obtained from simulations of the particle velocity $\frac{\partial u}{\partial t}$ on $\Gamma$, as supplied by the work group of $W$. Kropp at the Chalmers University in Gothenburg within the LeiStra3 cooperation and then converted from frequency to the time domain, see [2] for details.

In this experiment we consider the tire centered above $x=y=0$, elevated $2.1 \mathrm{~cm}$ above the street. In our units with the speed of sound $c=1$, we choose $\Delta t=0.01$, so that $\Delta t / h \approx 0.2$ and solve the integral equation (2.7) of the second kind for both Dirichlet and Neumann boundary conditions on $\partial \mathbb{R}_{+}^{3}$. The density is plotted in snapshots at the time steps 100, 200 and 300 in Figure 5.10 (for the Dirichlet problem). See $[2,8]$ for similar density profiles for the Neumann problem. Figure 5.8 shows the resulting sound pressure in the point $(2.8 \mathrm{~m}, 0,1.0 \mathrm{~m})$. The influence of the boundary conditions is clearly observed once the reflected wave has reached the point of observation, especially in the transient dynamics for short times. For long times, the Dirichlet conditions show a persistent oscillation of period around $7 \Delta t$. Figure 5.11 shows the absolute value of the Fourier transform of the sound pressure from Figure 5.8 for times $\geq 5.145$. The oscillations in time for the Dirichlet problem clearly manifest themselves as a broad peak around frequency $4800 \mathrm{~Hz}$, in physical units. For the Neumann problem a smaller resonance may be noticed around $1000 \mathrm{~Hz}$. In [2] we showed that such broad-band frequency results agree and are competitive with direct computations in frequency domain for passenger car and truck 


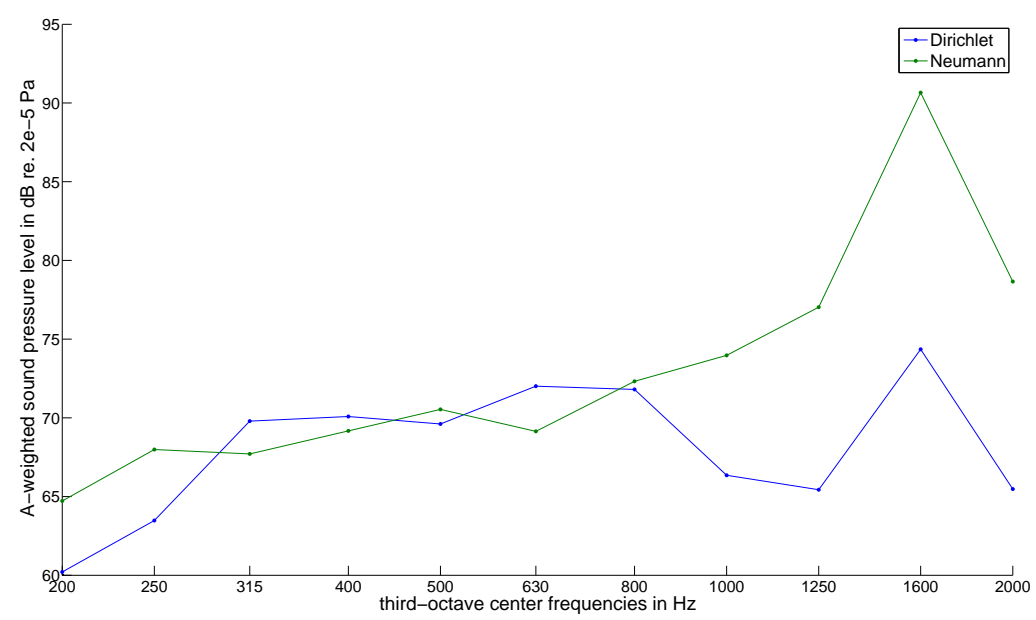

Fig. 5.12.: A-weighted sound pressure for Dirichlet and Neumann conditions, averaged over 321 points.

tires $1 \mathrm{~mm}$ above the street over a sound-hard street; they qualitatively agree with experiments.

In practice, it is often average characteristics and the human perception of the sound emission that are of interest. Figure 5.12 depicts an average over 321 points on the hemisphere $\left\{x \in \mathbb{R}_{+}^{3}:\|x\|_{2}=2\right\}$ of emission spectra like in Figure 5.11, also averaged over bands of frequencies. Here the A-weighted sound pressure level is plotted for frequencies up to $2000 \mathrm{~Hz}$, which provides an approximation to the human perception of noise. We observe that Dirichlet and Neumann conditions lead to similar average noise emission for frequencies between 300 and $800 \mathrm{~Hz}$. For higher frequencies, the noise level is significantly higher in the Neumann case, reflecting the resonance already observed in Figure 5.11. The possibility of such analyses gives time-domain boundary element methods a role in the study of traffic noise.

Acknowledgments. Parts of this work were funded by BMWi under the project SPERoN 2020, part II, Leiser Straßenverkehr, grant number 19 U 10016 F. H. G. acknowledges support by ERC Advanced Grant HARG 268105. C. O. is supported by a scholarship of the Avicenna foundation.

\section{References}

[1] A. Bamberger, T. Ha Duong, Formulation variationnelle espace-temps pour le calcul par potentiel retard de la diffraction d'une onde acoustique, Math. Meth. Appl. Sci., 8 (1986), 405-435 and 598-608.

[2] L. Banz, H. Gimperlein, Z. Nezhi, E.P. Stephan, Time domain BEM for sound radiation of tires, Comput. Mech., 58 (2016), 45-57.

[3] M. Costabel, Time dependent problems with the boundary integral equation method. In Encyclopedia of Computational Mechanics, E. Stein, R. de Borst, and J. R. Hughes, Eds. John Wiley \& Sons, Chichester, 2004, 703-721.

[4] T.A. Cruse, F.J. Rizzo, A direct formulation and numerical solution of the general transient elastodynamic problem I, J. Math. Analysis Appl., 22 (1968), 244-259.

[5] Y. Ding, A. Forestier, T. Ha Duong, A Galerkin scheme for the time domain integral equation of acoustic scattering from a hard surface, J. Acoust. Soc. Am., 86 (1989), 1566-1572. 
[6] O. von Estorff et al., Abschlussbericht SPERoN 2020, Teil II, Entwicklung eines performanten Rechenmodells zur Berechnung und Reduzierung der Geräuschabstrahlung von Reifen, 2014.

[7] M. Friedmann, R. Shaw, Diffraction of pulses by cylindrical obstacles of arbitrary cross section, J. Appl. Mech., 29 (1962), 40-46.

[8] H. Gimperlein, M. Maischak, E.P. Stephan, Adaptive time domain boundary element methods and engineering applications, Journal of Integral Equations and Applications, 29 (2017), 75-105.

[9] H. Gimperlein, Z. Nezhi, E.P. Stephan, A priori error estimates for a time dependent boundary element method for the acoustic wave equation in a half-space, Mathematical Methods in the Applied Sciences, 40 (2017), 448-462.

[10] H. Gimperlein, C. Oezdemir, D. Stark, E.P. Stephan, A residual a posteriori error estimate for the time domain boundary element method, preprint.

[11] M. Gläfke, Adaptive Methods for Time Domain Boundary Integral Equations, Ph.D. thesis, Brunel University, 2012.

[12] T. Ha Duong, Equations Integrales Pour la Resolution Numerique des Problemes de Diffraction d'ondes Acoustiques dans $R^{3}$, Ph.D. Thesis, Paris VI, 1987.

[13] T. Ha Duong, On retarded potential boundary integral equations and their discretizations, in: Topics in computational wave propagation, pp. 301-336, Lect. Notes Comput. Sci. Eng., 31, Springer, Berlin, 2003.

[14] T. Ha Duong, B. Ludwig and I. Terrasse, A Galerkin BEM for transient acoustic scattering by an absorbing obstacle, Internat. J. Numer. Methods Engrg., 57 (2003), 1845-1882.

[15] M. Ochmann, Closed form solutions for the acoustical impulse response over a masslike or an absorbing plane, J. Acoust. Soc. Am., 129:6 2011.

[16] E. Ostermann, Numerical Methods for Space-Time Variational Formulations of Retarded Potential Boundary Integral Equations, Ph.D. Thesis, Leibniz Universität Hannover, 2009.

[17] S. Sauter, A. Veit, Adaptive time discretization for retarded potentials, Numer. Math., 132 (2016), $569-595$.

[18] F.-J. Sayas, Retarded Potentials and Time Domain Boundary Integral Equations: A Road-Map, Springer Series in Comp. Math. 50, Springer, 2016.

[19] I. Terrasse, Résolution Mathématique et Numérique des Équations de Maxwell Instationnaires par une Méthode de Potentiels Retardés, Ph.D. Thesis, École Polytechnique, Palaiseau, 1993.

[20] A. Veit, Numerical Methods for the Time Domain Boundary Integral Equations, Ph.D. Thesis, Universität Zürich, 2011.

[21] A.E. Yilmaz, J.M. Jin, E. Michielssen, Time domain adaptive integral method for surface integral equations, IEEE Trans. Antennas Propagation, 52 (2004) 2692-2708. 